\title{
El retorno de la consciencia en la filosofía de Nietzsche *
}

\author{
The return of consciousness in Nietzsche's philosophy.
}

\author{
Por: Pereyra Nouveliere, Santiago Agustin * \\ Universidad Nacional de San Juan (UNSJ) \\ E-mail: nouvelieresanty@gmail.com
}

Fecha de recepción: 13/01/2020

Fecha de aprobación: 01/07/2020

DOI: $10.30972 /$ ach.054418

\section{Resumen}

A lo largo de este ensayo se elaborara una reflexión sobre el concepto de la consciencia en Nietzsche, un concepto que no suele ser tan trabajado en comparación a otros aspectos del autor, centrándome en algunos tipos de consciencia que concibe el autor y cuáles son sus implicancias en un sistema filosófico tan complejo como este. Al comienzo del escrito se muestran algunos ataques realizado a este pensador, los cuales como se muestran están totalmente injustificados, con el fin de poder desacreditar esta filosofía tan innovadora e irrepetible en nuestros tiempos. Como sería imposible analizar todas las obras del autor sobre este concepto, los especialistas que los trabajaron, y sus consecuencias en nuestro pensar. Solo se trabajaran brevemente ciertos parágrafos y apartados de Nietzsche, y se verá en particular su repercusión en ciertos sistemas que integran a la psicología.

\footnotetext{
* El presente trabajo surge en el marco del proyecto de investigación "El problema de la conciencia en las ciencias contemporáneas: nuevas líneas de investigación”. Resolución N²021/18-CS-UNSJ. Y continua lo trabajado en "Las implicaciones místico-metafísico entre la consciencia y el lenguaje", que está en vías de publicación en el libro de acta del: III Congreso Binacional de Investigación Científica y el VI Encuentro de jóvenes Investigadores.

* Estudiante avanzado de la licenciatura en Filosofía, de la Facultad de Filosofía, Humanidades y Arte (FFHA). Actualmente trabajando en el Instituto de Investigación de Filosofía (IDEF), dentro del proyecto de investigación mencionado, en la nota al pie de página anterior.
} 
Palabras claves: consciencia; ficción; Nietzsche; nihilismo; psicología.

\section{Abstract}

Throughout this essay, a reflection on the concept of consciousness in Nietzsche will be elaborated, a concept that is not usually so elaborated in comparison to other aspects of the author, focusing on some types of consciousness that the author conceives and what their implications are in a philosophical system as complex as this. At the beginning of the writing, some attacks on this thinker are shown, which as shown are totally unjustified, in order to discredit this innovative and unrepeatable philosophy in our times. As it would be impossible to analyze all the author works on this concept, the specialists who worked on them, and their consequences on our thinking. Only certain paragraphs and sections of Nietzsche will be briefly worked on, and its repercussion in certain systems that integrate psychology will be seen in particular.

Key words: consciousness; fiction; Nietzsche; nihilism; psychology.

\section{Cómo citar este artículo:}

APA: Pereyra Nouveliere, S. A. (2020). El retorno de la consciencia en la filosofía de Nietzsche, Acheronta, 5, 156-155. Recuperado de: (agregar dirección web)

En este artículo, se trabajara la concepción de la consciencia en Nietzsche comparándolo, o dialogando, con diversos autores mostrando ciertas semejanzas como divergencias. Algunos pensadores que han trabajado este concepto, los cuales estarán presente con mayor profundidad en el escrito, son por ejemplo: Ganni Vattimo, Sigmund Freud, Carl Gustav Jung, entre otros. Carecería de sentido la existencia de este ensayo, si solo se desarrollara cosas ya trabajadas y realizadas en otros autores. Por ello este artículo pretende 
Abril 2020.

incorporar una perspectiva más filosófica, a una problemática y a un concepto, que hoy en día suele ser asimilado o mayormente trabajado dentro del campo de la psicología.

Observando el contexto y la vida de Nietzsche podemos vislumbrar a lo largo de sus obras ciertas concepciones de la consciencia ${ }^{1}$, las cuales no necesariamente aparezcan con ese nombre ni tampoco sean mantenidas igualmente a lo largo de sus escritos. Es por ello que me surgen las siguientes preguntas o inquietudes: ¿Qué tipos consciencia existen en Nietzsche? o ¿Cuáles son los modos en que se manifiesta la consciencia en Nietzsche? Antes de proseguir, es necesario aclarar que no se trabajara este concepto en todas las obras de este pensador, sino que solo se abordará bajo determinadas obras. Primero se analizara el papel de la consciencia en "El origen de la tragedia", luego se pasara al papel de la consciencia en "Así hablo Zaratustra", después se verá el papel de la consciencia en "Más allá del bien y del mal", finalmente se dará una imagen conceptual general. Con ello no se sugiere hacer una investigación exhaustiva, sino que se busca precisar lo desarrollado al otorgar una o dos características de la consciencia por obra.

Este ensayo posee un recorrido interno, o un hilo de Ariadna, que se desarrollara de la siguiente manera: primero se detallaran ciertos prejuicios contra el autor, después se tomara lo desarrollado por Jung y Vattimo con respecto a la consciencia en Nietzsche, luego se analizara el papel de la consciencia en ciertas obras de Nietzsche, se proseguirá brindando una imagen general de la consciencia, terminando con unas reflexiones finales sobre lo trabajado.

En un primer acercamiento del pensador, antes de entrar a sus trabajos, encontramos el círculo de personas con las cuales se relacionó e influyeron de

\footnotetext{
${ }^{1}$ Cuyas divergencias etimológicas y conceptuales con el término conciencia, ya fueron analizadas en el trabajo denominado "Las implicaciones místico-metafísico entre la consciencia y el lenguaje".
} 
cierta manera en sus manuscritos. Para analizar, aunque sea brevemente, esta faceta particular de su pensamiento cabe destacar su relación con el medico Josef Breuer. Esta relación de médico-paciente se desarrolla entre sus escritos de "Más allá del bien y del mal" y "Así hablo Zaratustra", el cual durante sus conversaciones influyo en sus teorías con relación de los consciente. Dicho medico fue mentor y propulsor de las bases metodológicas para Sigmund Freud, otro psicólogo importante que leerá e interpretara de un modo distintos estos escritos es Carl Gustav Jung².

Esta influencia de Nietzsche podemos encontrarlo claramente en las actas de la asociación psicoanalítica de Viena, el primero de abril ${ }^{3}$. Durante dicha acta se presenta una discusión sobre la tercera parte en la obra "Genealogía de la moral". En la cual el psicólogo Sadger realiza la siguiente hipótesis ${ }^{4}$ sobre el estado psicológico de Nietzsche:

"En lo que atañe a Nietzsche, todavía no se puede tratar el tema, ya que aún no se han publicado los datos más importantes. Su madre no padecía de taras hereditarias, si algunos parientes, pero Nietzsche es sin duda el ejemplo típico de un sujeto con estigmas hereditarios. Además, hay en él un fuerte elemento de histeria; los estados epileptoides sin pérdida de conciencia, que el mismo dice haber sufrido durante su infancia, eran sin dudas síntomas histéricos" (Nunberg, 1979, 361)

Esta cita contiene un gran prejuicio contra este autor, este tipo de opiniones no son nada nuevo, ya abundaban en sus primeros escritos entre sus coetáneos. No hay que ser inocente al pasarlo por alto, porque el objetivo que se busca y se

\footnotetext{
2 Apare en su obra denominada "Tipos psicológicos", con su título original Psychologische Typen, la cual fue publicada en el año 1921.

${ }^{3}$ El acta numero cuarenta y cinco.

${ }^{4}$ Esta hipótesis de Sadger, se da en una discusión que mantiene con Adler, Graf, entre otros psicólogos. Sobre la cuestión mental de Nietzsche, la cual se encuentra registrada, en la misma acta que aparece mencionado en la nota al pie de página anterior.
} 
Abril 2020.

aspira es desestimar este pensamiento bajo estos argumentos u otros similares, sentenciándolo principalmente al crimen de la locura destruyendo todo posible respeto de sus escritos. De hecho este objetivo se cumplió durante un largo periodo, atrasando las lecturas y diversas traducciones de sus escritos. Un autor que anuncia brevemente esta problemática es Vattimo, en las siguientes líneas:

“el problema del origen sifilítico de esta locura no se ha podido resolver y volvió difícil la discusión filosófica sobre su obra al menos hasta la primera guerra mundial [....] Los teólogos [....] pensaban que su derrumbe psíquico podía haber tenido por causa una fallida superación del cristianismo y el consiguiente mantenimiento del irreprimible deseo de encontrar de nuevo a Dios" (Vattimo, 1996, 153)

Otro de estos estigmas más contemporáneo nuestro es el que se le dio después de la segunda guerra mundial, es decir la categoría de antisemita e ideólogo de la Alemania Nazi. Categoría que todo biógrafo y lector de Nietzsche ha descartado completamente, por pruebas irrefutables tanto en sus textos como en sus cartas o diarios, otorgándole esta prostitución de su pensamiento hacia las políticas fascistas a su hermana ${ }^{5}$. Valga la salvedad que todas las críticas fueron hechas a las enfermedades que padeció, aunque no se las realizaron a su sistema filosófico ni a su coherencia en su pensamiento plasmado en sus escritos, este modo de desacreditación es lamentablemente muy común en la historia de la filosofía. Un ejemplo concreto que pueda mencionarse, es el de Georg Hegel ya que fue muy criticado por apoyar al reinado de Prusia cuando en su juventud criticaba dicho gobierno, a su vez su

\footnotetext{
${ }^{5}$ Como bien lo explica el especialista Germán Cano, la manipulación de las obras de Nietzsche por parte de Elisabeth Forster-Nietzsche: "Y ella, ignorando pasajes contra el antisemitismo o promoviendo directamente la falsificación de textos y documentos de su hermano, será la principal responsable de poner esta obra al servicio de la propaganda nacionalsocialista." (Nietzsche, 2014, XXIV)
} 
pensamiento filosófico es sistemáticamente perfecto bajo su lógica y coherencia propia.

Viendo todo estas acusaciones que se le hizo para que no se leyeran sus textos podemos comprender sus palabras a través de la voz de Zaratustra "No me entienden: no soy la boca para estos oídos" (Nietzsche, 2014, 27), o también como lo anuncia en su tan conocido parágrafo 125 de la gaya ciencia (el discurso del loco o el hombre frenético):

"Llego demasiado pronto - dijo entonces- mi tiempo todavía no ha llegado. Este enorme acontecimiento aún está en camino y deambula aún no ha penetrado en los oídos de los hombres" (Nietzsche, 2014, 440)

Una vez hecho estas salvedades importantes para poder realizar un primer acercamiento a las obras de dicho pensador, vale aclarar lo complicado que es poder interpretar sus escritos y lo propenso que es llevarlos a diversas tergiversaciones, el fundamento de esto se haya en su modo de escritura: aforismos, metáfora, ironías, sarcasmo, prosa en poema largo, entre otros. En este sentido sus escritos tienen un tinte bastante oscuro, por ello hay que tener especial cuidado al interpretar sus trabajos, y poder realizar la necesaria división: entre lo que dice el mismo Nietzsche y lo que uno puede interpretar u analizar de él.

Habiendo dicho eso, me gustaría comenzar a analizar lo que concibe como la consciencia, cuyo concepto lo podemos encontrar perfectamente elaborado en las siguientes palabras:

"la conciencia es el campo donde luchan distintas partes del yo, sin que sea nunca posible decir cuál de estos yoes distintos es el auténtico [...] El yo solo es el escenario donde se desarrolla el drama de la vida moral, 
donde combaten impulsos distintos y opuestos [...] la conciencia está hecha, está producida, y por lo tanto no es una instancia última" (Vattimo, $1996,73)$

Dentro de esta cita se encuentran una definición de la consciencia con muchas similitudes a la que tiempo después construirá Freud ${ }^{6}$ con el psicoanálisis, aunque ambas poseen grandes e importantes diferencias. En el segundo apartado del texto anteriormente transcripto, se puede ver al yo como producto de dos impulsos opuestos, los cuales podrían ser llamados por lo menos en sus escritos de juventud como lo apolíneo y lo dionisiaco ${ }^{7}$.

Una interpretación que se puede dar de estos conceptos, distintos y aparentemente opuestos ${ }^{8}$, dentro del contexto del yo es la psiquiatría. En la que se puede observar como en ciertas patologías, se tiende a dos impulsos que dominan momentáneamente, uno que despierta, energética e incluso a veces tiende hacia la violencia (dionisiaco). Otra en cambio produce los efectos contrarios: tranquiliza, calma y de quietud (apolíneo) ${ }^{9}$.

El último apartado pero no por ello menos importante de este fragmento, habla de una consciencia producida y solo como una etapa más del proceso, este producto se realizara a través del lenguaje como herramienta por ello se realizara con una consciencia filológica. En la cual tanto el lenguaje como el

\footnotetext{
${ }^{6}$ Vale aclarar que Freud rechaza tajantemente cualquier tipo de influencia de esta filosofía, así lo deja expresado en las actas: "No conoce la obra de Nietzsche; sus ocasionales intentos de leerla fueron sofocados por un exceso de interés" (Nunberg, 1979, 363).

${ }^{7}$ Estos conceptos aparecen en su libro denominado, el origen de la tragedia, dentro del cual los define como impulsos cada uno con una característica particular: "el sueño y la embriaguez son fenómenos fisiológicos en los que puede apreciarse un contraste análogo al existente entre lo apolíneo y lo dionisiaco" (Nietzsche, 2014, 21-22)

${ }^{8}$ Son aparentemente ya que si uno se pone a estudiar esta obra con profundidad se puede notar que conservan variadas similitudes y entre ellos se necesitan, como afirma Vattimo "Apolo habla la lengua de Dionisio" (Vattimo, 1996, 132).

${ }^{9}$ Esta pelea de pulsiones también puede leerse en Freud, con la pulsión de vida (Eros) y la pulsión de muerte (Thánatos), con la que guardan muchas similitudes a lo propuesto por Nietzsche. A su vez también poseen similitudes con el pensamiento de Hegel (el cual influyo mucho en Nietzsche durante el periodo en que escribió esas teorías en su libro) y con Kant (que aparece sobre en su obra "Hacia la paz perpetua").
} 
intelecto se mantienen como ficciones necesarias para la vida, expresándolo con claridad en estas palabras: "El intelecto, como un medio para la conservación del individuo, desarrolla sus fuerzas capitales en la ficción" (Nietzsche, 2014, 350).

Para terminar esta parte se habla solo como una etapa, por ello no va a contener ningún ideal metafísico, también al concebirlo como ficción se distanciara bastante del psicoanálisis tanto en su percepción y en su función. Para poder completar esta idea, de la consciencia como una etapa (mas no por ello se culmina ahí), por ello podemos deducir que necesariamente lo consciente será lo que afirma el devenir y por lo tanto también el cuerpo, mientras que lo que niegue este devenir formara parte del inconsciente.

La función de la consciencia poseerá una doble finalidad, la primera que se ha dicho con anterioridad será la de su propia conservación, y la segunda una autoconsciencia de su propia ficción. Ya como primer paso para dirigirse al Übermensch es necesario poder ser capaces de despertar de nuestro sueño dogmático y poder afirmar tanto su propio limite como las ficciones en las que él vive, en otras palabras poder pasar por las dos anteriores transformaciones ${ }^{10}$. Por esto si bien será una ficción, pero no por esto una cualquier sino que será una ficción importante, así como también los son: el eterno retorno, la voluntad de poder, nihilismo, entre otros.

Ahora bien ya se mencionaron más de un tipo de consciencia, por lo que vale rescatar las preguntas que se elaboraron al inicio del trabajo, ¿Qué tipos consciencia existen en Nietzsche? o ¿Cuáles son los modos en que se manifiesta la consciencia en Nietzsche? Algunos nombres que le asigna a la consciencia con distintas funciones son la: filológica, histórica, moral, individual, grupal,

\footnotetext{
${ }^{10}$ Cuyas trasformaciones aparecen explicadas en las figuras del camello, león y niño; los cuales aparecen en la obra "Así hablo Zaratustra", dentro del apartado titulado "De las tres transformaciones".
} 
Abril 2020.

científica, tiempo ${ }^{11}$, etcétera. Así como de un instrumento musical puede sacarse muchos tonos y piezas distintas, igualmente de un solo concepto como este se aplica en varios particulares. Uno de estas manifestaciones de la consciencia, es la Nietzsche le dedica segmentos de sus obras y libros enteros, en otras palabras se está hablando de la consciencia moral. Niemeyer realiza una importante aclaración a esta consciencia complementada con la culpa judeocristiana:

"Un segundo planteamiento trata de aclarar la conciencia de culpabilidad: los «antiguos» no veían que hubiese «una «relación adecuada» entre culpa e infelicidad» sino que conocían todavía "la infelicidad inocente»; «solo con el Cristianismo se convierte todo [...] en castigo bien merecido» ( $M$ 78). N. reconoce la raíz de esta situación en la gran disparidad de poder entre el juez supremo (Dios) y los aspirantes potenciales a jueces" (Niemeyer, 2012, 124)

Este particular tipo de consciencia moral la cual se centra solamente en lo que se puede ganar o perder en un estadio metafísico, como una especie de economía del mercado místico, es fuertemente criticado en todo su sistema de pensamiento. Al centrarse solamente en la otra vida y no tanto en esta, ya que cuando se centra en esta es en modo de preparativo para la próxima, bajo este panorama Nietzsche utiliza su concepto amor fati. Cuyo concepto funcionara de contrapeso a esta moral, pues este amor es amar y aceptar la vida que se vive en el hoy, es aceptar el sufrimiento pero no porque en otra vida ganaremos algo sino porque en la vida misma hay sufrimiento. Otra de estas críticas es el desprendimiento y desprecio hacia el cuerpo, por ello no acepta el

\footnotetext{
${ }^{11}$ Este tipo consciencia lo nombra brevemente en una de sus correspondencias: "Tal vez yo busco, por un momento, abstraerme de la conciencia de tiempo, de la simpatía subjetiva y espontánea por Prusia" (Nietzsche, 2012, 412)
} 
devenir, como consecuencia está en un inconsciente o si se quiere en una consciencia falsa. La bifurcación de las personas que poseen este tipo de consciencia y la consciencia que aspira Nietzsche, se puede ver reflejado en el "Así hablo Zaratustra" dentro del apartado llamado "De los predicadores de la muerte", en específico dentro de estas líneas:

“iLes gustaría estar muertos, y nosotros deberíamos aceptar su voluntad! [...] Pero solo ellos han quedado refutados, y sus ojos, que solo ven un rostro en la existencia [...] Por todas partes resuena la voz de quienes predican la muerte: y la tierra está lleno de aquellos a quienes se les debe predicar la muerte. O la vida eterna: eso me da igual" (Nietzsche, 2014, 58 -59)

Aquí podemos vislumbrar como estos de la consciencia moral son estos predicadores de la muerte, o en otras palabras como menciona el texto en referencia a lo que siempre buscan y por ello no viven la vida, de la vida eterna. Mientras que el mismo Zaratustra será quien tenga la voz de la consciencia como ficción, de la vida consciente de la ficción, y por ello le permitirá vivir en la vida. Otra de las consecuencias de esta moral, es el reservarse y guardarse todos los impulsos (tanto positivos como negativos), una decisión fatal ya que en el momento que salgan estos impulsos será peor al haber estado contenida tanto tiempo, cuestión que plantea Jung bajo la lectura de Nietzsche:

"Las fuerzas impulsivas estancadas en el hombre civilizado son enormemente destructivas y mucho más peligrosas que los impulsos del primitivo, el cual vive constantemente en medida modesta sus impulsos negativos" (Jung, 1994, 173) 
Para poder finalizar este breve ensayo, sobre la consciencia en Nietzsche, con algunas de sus repercusiones. Habiéndolo trabajado en conjunción con diversos autores pertenecientes tanto a las disciplinas de la filosofía, como Vattimo, y de la psicología, como Freud o Jung, con la seriedad analítica para garantizar una hermenéutica valida dentro un sistema tan complejo. Cuyas vertientes, como se pudo observar a lo largo de este escrito, para que posea una interpretación enriquecedora y superadora, deben ser entendidas una en aproximación a la otra. Ya que ambas poseyeron, y poseen en la actualidad, una gran cantidad de problemáticas, concepto, métodos; salvaguardando las diferencias, poseen grandes puntos $u$ encuentros en común. También en ambas disciplinas, el pensamiento de Nietzsche tuvo grandes aportes en estos lugares en común que poseen, modificando e impulsando a construir nuevos sistemas filosóficos y psicológicos.

Es necesario rescatar, antes de terminar este trabajo, nuevamente las preguntas que se realizaron al comienzo y a la mitad del escrito ¿Qué tipos consciencia existen en Nietzsche? o ¿Cuáles son los modos en que se manifiesta la consciencia en Nietzsche? Lo cual podríamos responder, según lo trabajado aquí, que solamente existe una consciencia verdadera, real, que nos permitirá vivir la vida. Cuya consciencia, tendrá diversos modos de manifestarse, según la obra en la que aparezca del autor, en el momento de su vida, o la función que esté cumpliendo, ya sea para afirmar o criticar una idea. Esta misma diversidad de manifestaciones, fue ricamente aprovechada, por los diversos autores y escuelas que se han ido abordando.

Antes de finalizar, seria grato, el poder proponer una nueva perspectiva de investigación que me suscito en este escrito. Investigar a la consciencia en Nietzsche, en relación con la culminación del proceso que se da en el nihilismo, en otras palabras estar completamente conscientes de nuestras ficciones. Si tuviese un papel principal en este proceso, sería una piedra angular para poder 
reinterpretar a la consciencia en Nietzsche, que en la mayoría de las veces es socavada por su enorme profundidad, y su gran riqueza interna que posee, en su peculiar filosofía.

\section{Bibliografía}

Deleuze Gilles (2000): “Nietzsche”, Madrid, España, Arena Libros.

Fink Eugen (2000): "La Filosofía de Nietzsche", España, Alianza Universidad.

Jung Carl Gustav (1994): "Tipos psicológicos", España, Edhasa.

Niemeyer Christian (2012): “Diccionario Nietzsche”, España, Biblioteca Nueva.

Nietzsche Friedrich (2012): “Correspondencia I. Junio 1850- Abril 1869", España, Trotta.

Nietzsche Friedrich (2014): "Friedrich Nietzsche I", España, Gredos.

Nietzsche Friedrich (2014): "Friedrich Nietzsche II", España, Gredos.

Nietzsche Friedrich (2014): "Friedrich Nietzsche III", España, Gredos.

Nunberg Herman, Federn Ernst (1979): "Las reuniones de los miércoles. Actas de la Sociedad Psicoanalítica de Viena", Buenos Aires, Nueva Visión.

Perry Pinchas (2007): "El día que Nietzsche Iloro", Bulgaria, First Look International.

Safranski Rüdiger (2002): "Nietzsche. Biografía de su pensamiento", España, Tusquets Editores.

Vattimo Gianni (1996): “Introducción a Nietzsche”, Barcelona, España, Ediciones Península. 